\title{
The trigger logic of EUSO-SPB and its performance
}

\author{
J. Bayer \\ University of Tübingen, Kepler Center for Astro and Particle Physics, Tübingen - Germany
}

\section{Bertaina*, F. Fenu, M. Mignone, H. Miyamoto, K. Shinozaki}

Department of Physics, University of Torino \& INFN Torino - Italy

E-mail: bertaina@to.infn.it

\section{A. Cummings, J. Eser}

Department of Physics, Colorado School of Mines, Mines - US

\section{A. Jung}

APC, Univ Paris Diderot, CNRS/IN2P3 - France

\section{for the JEM-EUSO Collaboration}

EUSO-SPB (Extreme Universe Space Observatory - Super Pressure Balloon) is the first pathfinder mission of the JEM-EUSO program which hosts a trigger logic following the concept envisaged for the main mission. Prior to its implementation the logic has been tested offline by means of dedicated simulations and experimental data taken at the TurLab facility as well as by the previous path-finder missions EUSO-Balloon and EUSO-TA. All these tests allowed optimizing the performance of the algorithm, especially in terms of controlling the rate of fake events due to other types of luminous phenomena such as anthropogenic lights, airplanes, lightnings, meteors, etc. Due to the necessity of limiting the logic to a single level trigger, quite some efforts have been devoted to its codification and implementation in the Photo-Detector Module of EUSO-SPB which is the main board exploiting the trigger logic and controlling the front-end electronics. The performance of the implemented logic has been then verified by means of dedicated tests carried out in a field campaign at the Telescope Array site where the EUSO-SPB detector was exposed to the night sky luminosity and to a variety of point-like and diffused light sources such as stars, meteors, airplanes and lightnings. Moreover, dedicated tests were performed using the Central Laser Facility of Telescope Array and a mobile laser system of the Colorado School of Mines with variable intensity to mimic up-going cosmic ray showers and determine experimentally the triggering performance of EUSO-SPB. The trigger logic scheme and its hardware implementation as well as the results of the field tests carried out at the Telescope Array site in September-October 2016 and of the preliminary analyses of the data collected during EUSO-SPB flight are summarized.

35th International Cosmic Ray Conference - ICRC2017

10-20 July, 2017

Bexco, Busan, Korea

* Speaker. 


\section{Introduction}

EUSO-SPB [1] is a path-finder mission within the JEM-EUSO program with the aim of detecting for the first time from the edge of space Ultra-High Energy Cosmic Rays by means of the fluorescence technique. EUSO-SPB launched from Wanaka New Zealand on April 25th on a NASA Super Pressure Balloon and flew 12 days and 4 hours before sinking in the South Pacific ocean on May 6th. During the flight EUSO-SPB collected around hundred thousands events. Most of them were successfully downloaded on ground and are being processed. EUSO-SPB mounted on-board an autonomous First Level Trigger (FLT) similar to the one foreseen for JEM-EUSO [2]. Prior to its flight, EUSO-SPB was tested on a ground campaign in Utah, at the TA-EUSO [3] site where the detector was exposed to the night sky luminosity and to a variety of point-like and diffused light sources such as stars, meteors, airplanes and lightnings with the aim of understanding its performance. The Focal Surface (FS) of EUSO-SPB was based on MAPMTs (Hamamatsu Photonics R11265-03-M64) which have $8 \times 8$ pixels and for readout purposes $2 \times 2$ MAPMTs are grouped into one Elementary Cell (EC). The FLT works at the level of these ECs. Nine ECs form one Photo-Detector Module (PDM), which is the entire FS of EUSO-SPB and is the basic unit for the Second Level Trigger (SLT) [4].

\section{The First Level Trigger logic}

The FLT logic and first tests are described in [2]. The FLT rejects most of the fluctuations of the UV night-glow as well as electronic noise by requiring a locally persistent signal that lasts a few GTUs above the average background. In the FLT (see Fig. 1), pixels are grouped in cells of $3 \times 3$ pixels with one pixel overlap. The cells can not be shared by near-by MAPMTs. Therefore, one MAPMT hosts 36 cells. When a pixel in a cell detects a number of counts equal, or higher than, a preset threshold $N$, the FLT logic checks if for a certain number of following GTUs $(R)$ in a slot of consecutive GTUs $(P)$, there is at least one pixel in the same cell with an activity equal to, or higher than $N$, and the total number of photo-electrons integrated in the cell is higher than a preset value $S$. If this condition is verified a trigger is issued. $R$ and $P$ define the trigger mode suitable for the JEM-EUSO path-finder. In case of TA-EUSO or EUSO-SPB where the location of the Extensive Air Shower (EAS) can vary between 1 to $30 \mathrm{~km}, P$ values such as 0 or 1 are the most appropriate; consequently, $R$ can be 0 or 1. On the other hand, for JEM-EUSO, looking at $400 \mathrm{~km}$ distant EAS, $P=4$ and $R=2$. Having fixed, $P$ and $R$, the $N$ and $S$ parameters are set as a function of the average background level to keep the rate of triggers on false positives at $\sim 1 \mathrm{~Hz} / \mathrm{EC}$. The parameters can also be set via an external command. Even in the case of JEMEUSO where the Field-of-View (FoV) of one EC is much wider, an EAS is visible in one EC for less than 45 GTUs. This is much shorter than the minimum fraction of time that lightning (ms), meteors (hundreds ms) and cities/airplanes (seconds) will illuminate 1 EC. Starting from the GTU in which the FLT fires, a confirmation counter is activated. For a preset number of consecutive GTUs $\left(N_{G T U}\right)$, the confirmation counter is increased by 1 count for each GTU in which the FLT is fired. After $N_{G T U}$, if the confirmation counter has passed a certain threshold $N_{G T U}^{t h r}$, the trigger is not activated because it indicates that the FLT fired for a fraction of time that exceeded the expected duration of an EAS. Currently, $N_{G T U}=73$ and $N_{G T U}^{t h r}=72$. However, the two numbers can be varied 
independently. If the value accumulated in the confirmation counter is lower than $N_{G T U}^{t h r}$, the trigger confirmation is issued and the SLT is activated. The FLT passes to the SLT the information of time and location of the cells that were fired. The FLT parameters are set according to the average background level on the MAPMTs. Thresholds are set at MAPMT level. For each pixel an average background level is calculated every 128 GTUs $(320 \mu \mathrm{s})$. The pixel with the highest average count determines the thresholds for the entire MAPMT. This method takes more precisely into account the non-uniformity among pixels, as well as the presence of man-made sources with very limited spatial extension. All the artificial sources such as cities, planes, ships can be considered static at this level. The trigger logic for one EC as described previously has been implemented on a Xilinx
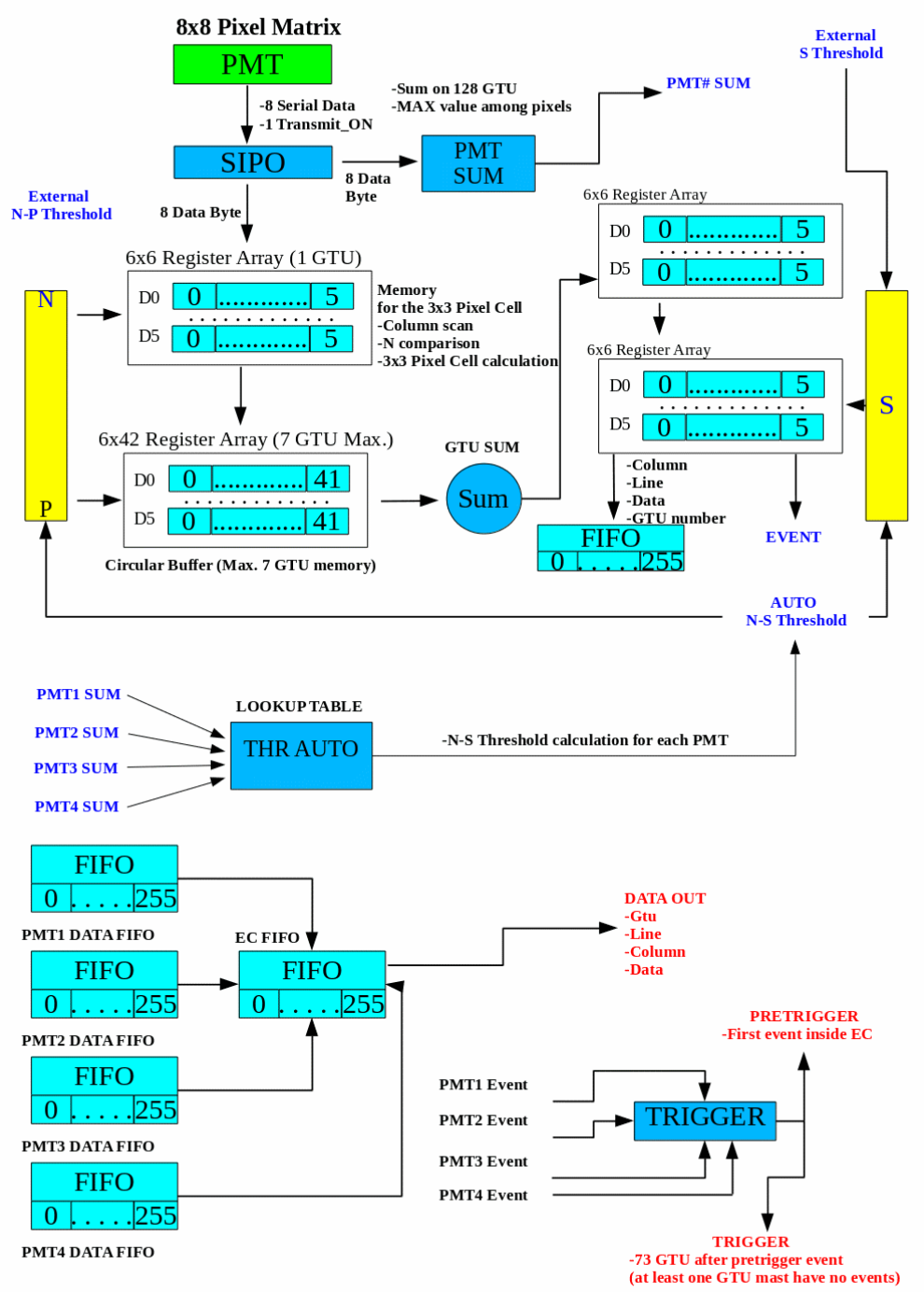

Figure 1: Schema of the VHDL implementation of the FLT logic.

\section{Virtex6 model XC6VLX240T [5] FPGA.}

To be able to implement the FLT for all nine ECs for EUSO-SPB, several changes to the PDM hardware were done with focus on the general VHDL implementation in the PDM FPGA. The PDM board for EUSO-SPB is an advanced version of the one which was developed for EUSO- 
Balloon. Details on the previous version of the hardware and the VHDL implementation can be found in [6]. The main advancements to the EUSO-SPB PDM board (shown in Fig. 2) are based

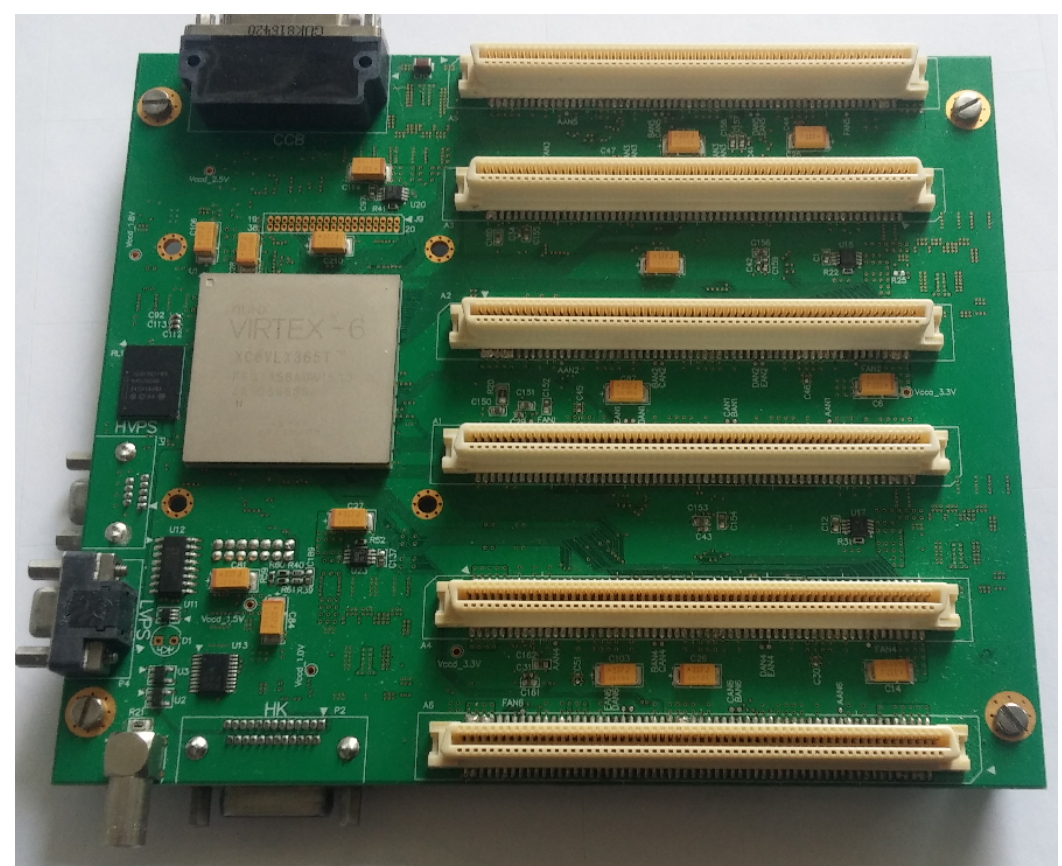

Figure 2: EUSO-SPB PDM board. The most visible changes in comparison to the EUSO-Balloon version is the different FPGA and the added holes around it to support the mounting of a special designed heat-sink. Other changes are not visible on this picture because the updated power-supply is mounted on the back side and the change in the grounding is on the internal layers.

on the experiences made during the development, integration and testing of the EUSO-Balloon hardware and can be summarized in two topics:

Power distribution and thermal improvements: To achieve a better power dissipation, the power distribution system which generates the various internal voltages for the FPGA and the periphery was completely re-designed and most of the voltage regulators were replaced by high efficiency switch mode regulators. To remove the dissipated power from the electronics in the low pressure environment during the balloon flight, a heat-sink was designed and mounted to the FPGA. In addition, the grounding scheme of the PDM-board and ASIC boards was redesigned which lead to an overall improvement of the electronic noise level.

VHDL implementation As the resources available in the XC6VLX240T were not enough to implement the FLT for all nine ECs together with all the glue logic around them, and the general control logic (like interfaces, data buffers, state-machines, etc.) the VHDL was re-written or improved to make use of the specific architecture of the Virtex-6. The tight schedule of the project made it necessary to develop the hardware and the VHDL in parallel, and therefore it was decided to exchange the FPGA by a pin-compatible one from the same family which has slightly more resources available - the XC6VLX360T. The final implementation of the FLT for all nine ECs including all the control logic and interfaces necessary and even additional functionality not available in the EUSO-Balloon flight uses now around $34 \%-43 \%$ of the available resources. 


\subsection{Hardware implementation of the FLT}

The basic scheme of the FLT is given in Fig. 1 and its main components are:

- Serial Pixel in to Column out (SIPO): This is the interface module towards the ASIC and receives the serial data from the single MAPMT channels, checks the data integrity and hands the pixel counts over to the next modules.

- PMT AVG: This module calculates the average of 128 GTUs which is used to estimate the current background level and to set the $\mathrm{N}$ - and S-thresholds accordingly.

- 6x6 Register Array (1 GTU): The received data is compared to the N-threshold and if above, the integration of the various $3 \times 3$ pixel boxes is performed.

- 6x42 Register Array (7 GTU Max.): This data buffer is used to apply the P- and Rthresholds together with the 'GTU SUM' module. In the current implementation it buffers the data for a maximum of 7 GTUs.

- 6x6 Register Arrays: The last two data buffers are used to compare the integrated 3x3 boxes to the $\mathrm{S}$ threshold and to store the information (position, integrated value and GTU number) to a FIFO and issue a pre-trigger.

- EC FIFO: This module simply collects the data from the single MAPMTs by reading them out in a round-robin fashion.

- TRIGGER: The trigger module finally collects the single cell triggers and checks that the pre-triggers are not lasting for more than 72 GTUs. This is done to remove triggers coming from slow and persisting events.

The main optimizations done in the implementation of the FLT for EUSO-SPB were on one hand the adaption of the VHDL to the specific hardware architecture of the Virtex-6 to make use of various dedicated hard-macros and on the other hand by doubling the internal clock speed and to serialize the processing of the FLT. This way, the resource utilization was greatly reduced via several steps. For example, due to the serialization of some processes and re-using the internal memories to total usage of the internal BRAM blocks was reduced by $\approx 85 \%$.

\section{The Utah Campaign}

In September 2015, a first test of the FLT was performed at EUSO-TA with the trigger logic implemented only on one EC of the EUSO-Balloon PDM board. Such test provided a first validation of the logic [6]. In October 2016, the fully assembled EUSO-SPB detector was tested at the EUSO-TA site to measure the overall response of the detector and to calibrate it by means of the Central Laser Facility (CLF) of Telescope Array and the portable Ground Laser System (GLS) of Colorado School of Mines. This time the FLT trigger was tested on the entire PDM for about a week. The trigger logic was set with $P=1$ and $R=1$. In normal sky conditions, the FLT rate was below $1 \mathrm{~Hz}$ on the entire PDM, increasing to few $\mathrm{Hz}$ during thunderstorms outside the FoV (the 
system could not have been operated with thunderstorms in the FoV) or during the passage of airplanes. This was due to the fact that the strobe lights of airplanes have very sharp raising phase of the signal, with longer time decay. Being just above threshold in some cases the FLT was not pre-triggered for $N_{G T U}^{t h r}$ GTUs, failing to recognize it as a slow event. The fact that in normal conditions stars were not creating huge bursts of triggers proved that the logic is capable of dealing with very slow but bright moving objects. Only rarely, when a bright star was passing through different MAPMTs and the light was scattered in an irregular way from the filters, a slight increase in the trigger rate was observed. Similar situation occurred in presence of meteoroids. A few of them triggered EUSO-SPB but at the level of no more than a few $\mathrm{Hz}$, which means that the logic successfully avoided too high trigger rates. It is worth remembering that the JEM-EUSO requirement on the FLT is to have a trigger rate $<7 \mathrm{~Hz}$ to make sure that the SLT is capable of processing the data on time. Most of the time, EUSO-SPB trigger logic was tested using CLF and GLS laser shots. On moonless nights and amenable weather the CLF, placed at a distance of $21 \mathrm{~km}$ from EUSO-TA and EUSO-SPB, fires 300 vertical laser pulses of $3 \mathrm{~mJ}$ and $355 \mathrm{~nm}$ at $10 \mathrm{~Hz}$ every half hour. The GLS laser is a mobile system which was set at a distance of $25 \mathrm{~km}$ from EUSO-SPB and EUSO-TA with a laser intensity in the range of 1 to $86 \mathrm{~mJ}$. As the laser itself is steerable, the geometry of the laser track can be varied more freely, mimicking EAS of different energy and direction. The GLS turned to be essential to provide a first estimation of the energy threshold of the FLT to trigger on EAS-like events. Left side of fig. 3 shows an example of a $2 \mathrm{~mJ}$ GLS laser event detected by EUSO-SPB. In this condition the trigger efficiency was $100 \%$.
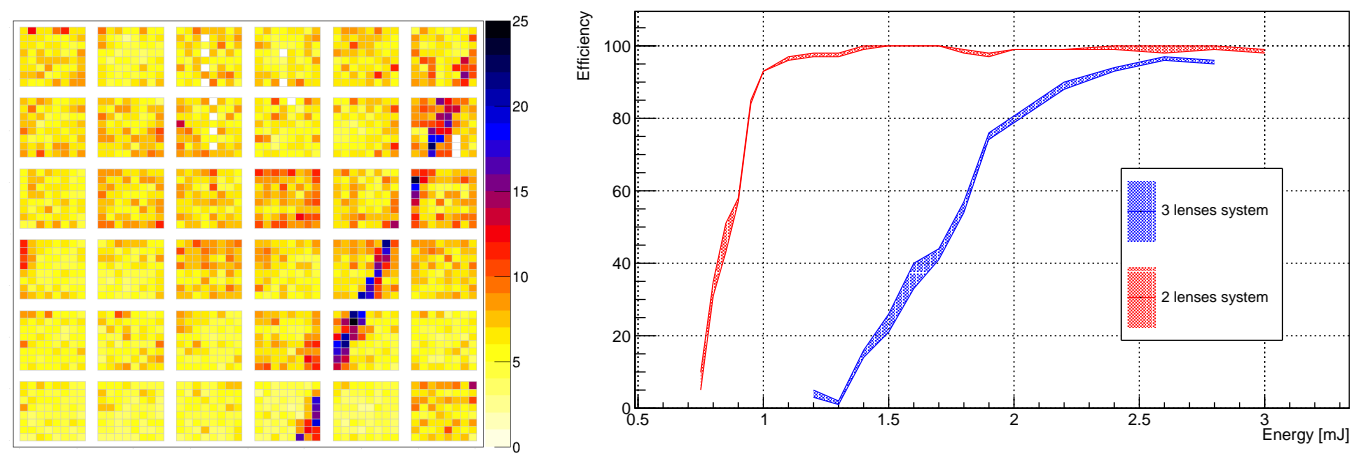

Figure 3: GLS laser event ( $2 \mathrm{~mJ}$ ) going through the FoV of the PDM adopting the 2 lens system (left) and trigger efficiency as a function of laser energy (right) with 2 and 3 lens system for vertical shots. The 3 lens system was only tested up to $2.7 \mathrm{~mJ}$.

This test was performed adopting two different lens configurations: a 2-lens system, without a diffractive lens in between which would correct the chromatic aberrations; a 3-lens system which is the basic configuration for JEM-EUSO. As swapping from one lens configuration could only be done in day time, the measurements were performed on different nights but with similar sky conditions to avoid major systematic effects in the comparison. Right side of fig. 3 shows the efficiency in triggering vertical laser shots in both conditions as a function of the laser energy.

The two-lens configuration turned out to be much better performing, despite a larger spot size on the focal surface. By comparing the laser signal on the FS and the expectation from simulations of EAS performed with ESAF and OffLine simulation codes, the result was that laser intensity 
giving $\sim 50 \%$ trigger efficiency is equivalent to that expected from $\sim 3 \times 10^{18} \mathrm{eV}$ and $45^{\circ}$ inclined EAS as seen from EUSO-SPB at an altitude of $33 \mathrm{~km}$. This was a positive result because it confirmed within 30-50\% uncertainty the energy threshold assumed for EUSO-SPB in simulations. More detailed information on the tests performed in Utah with EUSO-SPB can be found in [7].

\section{Trigger performance on flight}

On April 25th, EUSO-SPB was lifted by a Super Pressure Balloon of NASA and flew for about 12 days and operated during all nights without moon. Hundred thousands of packets of data were recorded. About $75 \%$ of them was downloaded before the flight terminated. Data were acquired in nominal runs of 120 seconds. Every hour a much shorter run (40 seconds) was started and data immediately downloaded to understand the general performance of the detector. Fig. 4 shows the overall trigger rate during the entire flight. In the figure, an indication of the trigger modes as a function of time is also indicated. For the first three nights EUSO-SPB operated in the $P=0$ and $R=0$ mode, which means single GTU operation. This was expected to be the most effective one to detect EAS. In normal condition the trigger rate was around $\sim 1 \mathrm{~Hz} / \mathrm{EC}$, however, this was too high for the downlink telemetry requirements. During all the rest of the flight, except for one night, the $P=1$ and $R=1$ mode, as used in Utah, was operated. In this case the overall trigger rate was below 2 $\mathrm{Hz} / \mathrm{PDM}$. The peaks above $10 \mathrm{~Hz}$ during the flight are often due to electronics disturbances on one specific EC. The night of May 5th, the $P=4$ and $R=2$ mode (JEM-EUSO trigger mode) was tested. The trigger rate was always below $2 \mathrm{~Hz} / \mathrm{PDM}$. In general, the FLT triggered within the JEM-EUSO
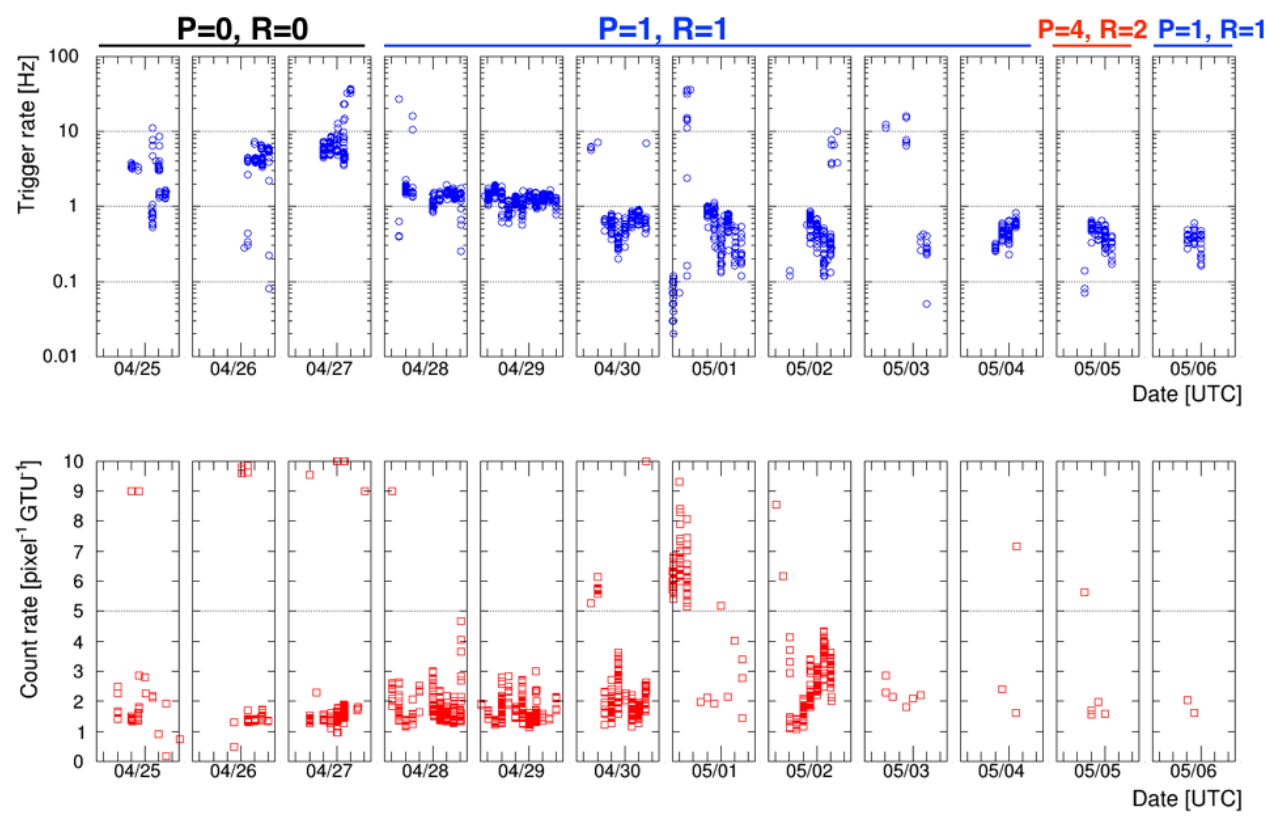

Figure 4: Top: EUSO-SPB trigger rate in flight. Bottom: average count rate measured at pixel level.

requirements (below $7 \mathrm{~Hz} / \mathrm{PDM}$ ). However, the trigger rate on flight was typically higher than what observed on ground. A preliminary analysis of the data seem to indicate that the periods with high 
trigger rate are related to electric noise. A certain number of events compatible with cosmic rays directly impacting in the detector have been recognized. Simulations are planned to understand and cross-check the rate. The analysis to search for EAS candidates has started. Simulations indicate that the number of expected events is of the order of 1-2 in the available data sample.

\section{Conclusions}

The FLT was tested for the first time on board a JEM-EUSO instrument. In general, its performance turned out to be within the requirements, even though for EUSO-SPB slightly lower trigger rate would have been more appropriate due to the telemetry bandwidth. The data collected with EUSO-SPB will be extremely important to test the performance of Mini-EUSO FLT logic which is slightly different from EUSO-SPB, to make sure that the trigger rate remains within the constraints imposed by the mission.

\section{Acknowledgments}

This work was partially supported by Basic Science Interdisciplinary Research Projects of RIKEN and JSPS KAKENHI Grant (22340063, 23340081, and 24244042), by the Italian Ministry of Foreign Affairs and International Cooperation, by the Italian Space Agency through the ASI INFN agreement n. 2017-8-H.0, by NASA award 11-APRA-0058 in the USA, by the Deutsches Zentrum für Luft- und Raumfahrt, by the Helmholtz Alliance for Astroparticle Physics funded by the Initiative and Networking Fund of the Helmholtz Association (Germany), and by Slovak Academy of Sciences MVTS JEM-EUSO as well as VEGA grant agency project 2/0132/17. We acknowledge support from French space agency CNES. Russia is supported by the Russian Foundation for Basic Research Grant No 16-29-13065-ofi-m. The Spanish Consortium involved in JEMEUSO is funded by MICINN \& MINECO under the Space Program projects: AYA2009-06037E/AYA, AYA-ESP2010-19082, AYA-ESP2011-29489-C03, AYA-ESP2012-39115-C03, AYA-ESP201347816-C4, MINECO/FEDER-UNAH13-4E-2741, CSD2009-00064 (Consolider MULTIDARK) and by Comunidad de Madrid (CAM) under projects S2009/ESP-1496 \& S2013/ICE-2822.

\section{References}

[1] S. Bacholle et al. (JEM-EUSO Coll.), "The EUSO-SPB Instrument", This Conference Proceedings, Busan (2017).

[2] G. Abdellaoui et al. (JEM-EUSO Coll.), Nucl. Instr. \& Meth. A 866 (2017) 150.

[3] J.H. Adams et al. (JEM-EUSO Coll.), Exp. Astronomy 40 (2015) 301.

[4] J. Bayer et al. (JEM-EUSO Coll.), Proc. 33th ICRC (Rio de Janeiro), \#0432 (2013); arXiv:1307.7071.

[5] http://www.xilinx.com/support/documentation/data_sheets/ds150.pdf

[6] A. Jung, JEM-EUSO prototypes for the detection of ultra-high-energy cosmic rays (UHECRs): from the electronics of the photo-detection module (PDM) to the operation and data analysis of two pathfinders, Thesis, 2017.

[7] J. Eser et al. (JEM-EUSO Coll.), "Preflight calibration and testing of EUSO-SPB in the lab and the desert", This Conference Proceedings, Busan (2017). 